OPEN ACCESS

Edited by:

Brahim Tabarki Melaiki,

University of Sousse, Tunisia

Reviewed by:

Fahad A. Bashiri,

King Saud University, Saudi Arabia

Erdem Tüzün

Istanbul University, Turkey

Ruzica Kravljanac,

The Institute for Health Protection of

Mother and Child Serbia, Serbia

*Correspondence:

Li Jiang

dr_jiangcqmu@163.com

Specialty section:

This article was submitted to

Pediatric Neurology,

a section of the journal

Frontiers in Pediatrics

Received: 04 July 2021 Accepted: 30 August 2021 Published: 01 October 2021

Citation:

Tan C, Jiang Y, Zhong M, Hu Y,

Hong S, Li X and Jiang L (2021) Clinical Features and Outcomes in Pediatric Autoimmune Encephalitis Associated With CASPR2 Antibody.

Front. Pediatr. 9:736035.

doi: 10.3389/fped.2021.736035

\section{Clinical Features and Outcomes in Pediatric Autoimmune Encephalitis Associated With CASPR2 Antibody}

\author{
Chengbing Tan ${ }^{1,2}$, Yan Jiang ${ }^{1,2}$, Min Zhong ${ }^{1,2}$, Yue Hu ${ }^{1,2}$, Siqi Hong ${ }^{1,2}$, Xiujuan $\mathrm{Li}^{1,2}$ and \\ Li Jiang ${ }^{1,2 *}$
}

\begin{abstract}
'Department of Neurology, Children's Hospital of Chongqing Medical University, National Clinical Research Center for Child Health and Disorders, Ministry of Education Key Laboratory of Child Development and Disorders, Chongqing, China,

${ }^{2}$ Chongqing Key Laboratory of Translational Medical Research in Cognitive Development and Learning and Memory Disorders, Chongqing, China
\end{abstract}

Background: Contactin-associated protein-like 2 (CASPR2) neurological autoimmunity has been associated with various clinical syndromes involving central and peripheral nervous system. CASPR2 antibody-associated autoimmune encephalitis is mostly reported in adults. Analysis of the clinical presentation and prognostic data of CASPR2 antibody-associated autoimmune encephalitis in children remains important.

Methods: A single-center retrospective review of children diagnosed with CASPR2 antibody-associated autoimmune encephalitis from June 1st, 2018 to October 31st, 2020.

Results: Six patients were identified. The median age was 12 years (range 1.8-14), with an overall male predominance of $83 \%$ (5/6). Commonest clinical features were psychiatric symptoms (6/6), movement disorders (4/6), altered consciousness (3/6), sleep disorders $(3 / 6)$, and headache (3/6). Four patients (4/6) received first-line therapy alone (steroids combined with intravenous immunoglobulins), and two patients $(2 / 6)$ received secondline therapy (rituximab, mycophenolate mofetil, or cyclophosphamide). All patients showed no peripheral nervous system involvement. One patient had comorbidities with systemic lupus erythematosus. No evidence of neoplastic disease was found in the whole cohort. All patients had favorable outcomes (modified Rankin Score 0-2) with recurrence rate at $0 \%$, respectively.

Conclusion: CASPR2 antibody-associated autoimmune encephalitis is rare in children. Our findings suggest that this type of encephalitis seems to occur more frequently in older children. Patients respond well to immunotherapy and usually demonstrate a favorable clinical outcome. Associated tumors are extremely rare.

Keywords: autoimmune encephalitis, contactin-associated protein-like 2, clinical features, outcomes, children

\section{INTRODUCTION}

Autoimmune encephalitis (AE) refers to a group of antibody-mediated inflammatory diseases of the brain. The major neurologic manifestations of AE include altered level of consciousness, confusion, sleep disturbances, movement disorders, seizures or autonomic dysfunction. To date, several antibodies against neuronal cell-surface or synaptic proteins have been identified (1). 
Voltage-gated potassium channels (VGKCs) are present on the membrane of neurons in both the central and peripheral nervous system which were initially detected in rare patients suffering from neuromyotonia (NMT) in 1995. Subsequently, VGKC antibodies were found in patients with Morvan syndrome (MoS) which mixes NMT and encephalopathy, and in patients with limbic encephalitis. Leucine-rich glioma-inactivated protein 1 (LGI1) and contactin-associated protein-like 2 (CASPR2) were identified as the main antigens within the VGKC in 2010 (2). CASPR2 is a membrane protein that is widely expressed in neurological sites such as the cortex, limbic system, basal ganglia and sensory organs. CASPR2-associated diseases mainly include gene-associated neurodevelopmental diseases and antibodyassociated autoimmune diseases. Mutations in the gene encoding CASPR2 have been reported in patients with autism spectrum disorder, epilepsy and other neuropsychiatric problems. The clinical spectrum of autoimmune diseases associated with antiCASPR2 antibody can manifest as encephalitis, NMT, MoS and painful neuropathy, and are mostly reported in adults (3). Pediatric manifestations of CASPR2 autoimmunity are less well studied and limited to single case reports or small case series (4). In the present study, we report 6 pediatric cases of CASPR2 antibody-associated autoimmune encephalitis.

\section{METHODS}

We retrospectively collected clinical data from patients with autoimmune encephalitis hospitalized at Children's Hospital of Chongqing Medical University, the largest tertiary pediatric medical center in southwest China, from June 1st, 2018 to October 31st, 2020. All the patients included in the study met the pediatric autoimmune encephalitis criteria as follows (5): (1) Onset of neurologic and/or psychiatric symptoms over $\leq 3$ months in a previously healthy child; (2) Two or more clinical features including altered mental status/level of consciousness or EEG with slowing or epileptiform activity, focal neurologic deficits, cognitive difficulties, acute developmental regression, movement disorder, psychiatric symptoms, seizures not explained by a previously known seizure disorder or other condition; (3) Two or more neuroinflammation features including CSF inflammatory changes (leukocytosis $>5$ cells $/ \mathrm{mm}^{3}$ and/or oligoclonal banding), MRI features of encephalitis, brain biopsy showing inflammatory infiltrates and excluding other disorders; (4) Presence in serum and/or CSF of well-characterized autoantibodies associated with AE; (5) Reasonable exclusion of alternative causes. Other etiologies such as infectious, metabolic, toxic and hereditary diseases were excluded based on clinical history and necessary auxiliary examinations.

Detailed clinical examination, imaging examination and cerebrospinal fluid (CSF) examination were used to confirm the diagnosis. Specimens were tested for CASPR2-IgG by a third-party clinical laboratory center. Sera or CSF Caspr2IgG were detected by a cell-based assay (CBA) using human embryonic kidney (HEK293T) cells transiently co-transfected with full-length human Caspr2 and pcDNA3.1-EGFP. After $36 \mathrm{~h}$ of transfection in 96-well plate, the cells were fixed with $4 \%$ paraformaldehyde for $20 \mathrm{~min}$, and ready for antibody detection. Sera were diluted at 1:10 in PBS-10\% goat serum and incubated on cells for $2 \mathrm{~h}$ at room temperature. Cells were then washed in PBS- $0.1 \%$ Tween 20 for 3 times, incubated for 30 min with goat anti-human IgG (1:500, Thermo Scientific), washed again in PBS-0.1\% Tween 20, and evaluated by immunofluorescence microscopy. Two independent masked assessors classified each sample as positive or negative based on the intensity of surface immunofluorescence in direct comparison with non-transfected cells and control samples. Once confirmed, the Caspr2+ sera were then serially diluted by three-fold from $1: 10$ to $1: 1,000$ to determine the titers. The final titer was defined as the sample dilution value for which specific fluorescence was barely but clearly identifiable and expressed as the corresponding dilution value. In parallel, specific antibodies including $\mathrm{N}$-methyl-d-aspartate receptor (NMDAR), $\alpha$-amino-3-hydroxy-5-methyl-4-isoxazolepropionic acid receptor (AMPAR), leucine-rich glioma-inactivated protein 1 (LGI1) and $\gamma$-aminobutyric acid type B receptor (GABABR) were evaluated. Autoimmune and tumor-related assessments included autoantibody series, antinuclear antibody profile, serum complement levels, thyroid function, Chest-abdomen computed tomography (CT)-enhanced scan, and tumor markers (human chorionic gonadotropin, carcinoembryonic antigen, neuron specific enolase, and vanillylmandelic acid). Necessary tests were performed to exclude infections, including cerebrospinal fluid culture and staining (bacterial, tuberculosis, and fungal), PCR and antibody testing of serum and cerebrospinal fluid (herpes simplex virus, Epstein-Barr virus, enterovirus, and Mycoplasma pneumoniae), and serological testing for HIV and syphilis. Patients with unexplained psychiatric symptoms were also tested for autoimmune encephalitis-related antibodies.

The modified Rankin Scale (mRS) score was used to retrospectively assess the neurological severity of the acute phase and the outcome of the final follow-up (6). The follow-up was conducted through outpatient visits or telephone interviews. Long-term good prognosis was defined as mRS score $\leq 2$, and long-term poor prognosis was defined as mRS score $>2$. Relapse was defined as recurrence of symptoms after full or partial recovery, with sustained improvement for at least 2 months (7).

Statistical analyses were performed using SPSS statistical software (IBM, New York, New York, 2019). Descriptive statistics were applied to analyze clinical data, such as medians and percentages.

\section{RESULTS}

\section{Demographic and Clinical Features}

Positive autoimmune encephalitis antibodies were detected in 103 of 674 suspected patients. Nine patients were positive for anti-CASPR2 antibodies. Two patients presented only with acute onset of psychiatric symptoms, which resolved spontaneously within 1 week without any treatment. Another patient presented with drowsiness and acute cerebellar ataxia, which was selflimited within 1 week without immunotherapy. Therefore, the above three patients with a serum antibody titer of 1:10 were 
TABLE 1 | Clinical and investigatory findings of patients with positive anti-CASPR2 antibody.

\begin{tabular}{|c|c|c|c|c|c|c|}
\hline No. & Age, Y/Sex & Clinical features & Cranial MRI & EEG & CSF & CASPR2-IgG titer \\
\hline 1 & $12.8 / \mathrm{F}$ & $\begin{array}{l}\text { Fever, headache, altered } \\
\text { consciousness, seizures, } \\
\text { psychiatric symptoms, facial } \\
\text { butterfly erythema, oral ulcer }\end{array}$ & Normal & $\begin{array}{l}\text { Slow background } \\
\text { activity (delta range); } \\
\text { epileptiform discharge }\end{array}$ & $\begin{array}{l}\text { WBC } 4 \\
\text { Prot } 41\end{array}$ & Serum 1:10 \\
\hline 2 & 11.6/M & $\begin{array}{l}\text { Headache, psychiatric } \\
\text { symptoms, sleep disorders, } \\
\text { movement disorders }\end{array}$ & Normal & Normal & $\begin{array}{l}\text { WBC } 11 \\
\text { Prot } 28\end{array}$ & Serum 1:30 \\
\hline 3 & 12.3/M & $\begin{array}{l}\text { Fever, headache, psychiatric } \\
\text { symptoms, sleep disorders, } \\
\text { hypertension, movement } \\
\text { disorders }\end{array}$ & Normal & Focal slow waves & $\begin{array}{l}\text { WBC } 14 \\
\text { Prot } 102\end{array}$ & Serum 1:10 \\
\hline 4 & $1.8 / \mathrm{M}$ & $\begin{array}{l}\text { Seizures, psychiatric symptoms, } \\
\text { movement disorders }\end{array}$ & Normal & Focal slow waves & $\begin{array}{l}\text { WBC } 2 \\
\text { Prot } 25\end{array}$ & Serum 1:10 \\
\hline 5 & $14 / \mathrm{M}$ & $\begin{array}{l}\text { Altered consciousness, } \\
\text { psychiatric symptoms, sleep } \\
\text { disorders, aphasia }\end{array}$ & Abnormal $^{\dagger}$ & $\begin{array}{l}\text { Slow background } \\
\text { activity (theta range) }\end{array}$ & $\begin{array}{l}\text { WBC } 0 \\
\text { Prot } 35\end{array}$ & Serum 1:10 \\
\hline 6 & $6.9 / \mathrm{M}$ & $\begin{array}{l}\text { Altered consciousness, } \\
\text { psychiatric symptoms, } \\
\text { movement disorders }\end{array}$ & Normal & $\begin{array}{l}\text { Slow background } \\
\text { activity (theta range) }\end{array}$ & $\begin{array}{l}\text { WBC } 0 \\
\text { Prot } 15\end{array}$ & Serum 1:30 \\
\hline
\end{tabular}

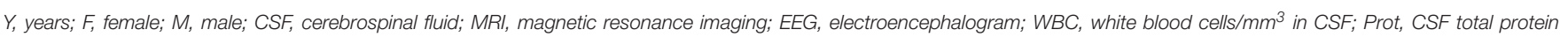
(mg/dl); CASPR2-lgG, contactin-associated protein-like 2 lgG.

${ }^{\dagger}$ Focal demyelination of the bilateral frontal lobes.

excluded from the present study. Six patients with positive antiCASPR2 antibody were diagnosed with autoimmune encephalitis and included in this study.

Demographic and clinical features are shown in Table 1. The median age was 12 years (range 1.8-14). The male to female ratio was 5:1. The initial symptoms were psychiatric symptoms $(6 / 6)$, frequent seizures (1/6), fever (2/6), and headache $(1 / 6)$. The common clinical manifestations were psychiatric symptoms $(6 / 6)$, movement disorders (4/6), altered consciousness $(3 / 6)$, sleep disorders $(3 / 6)$, headache $(3 / 6)$, fever $(2 / 6)$, seizures $(2 / 6)$, and speech disorders $(1 / 6)$ during the course of the disease. In this group of patients, the main psychiatric symptoms were hallucinations, babbling, aggressive behavior, disruptive behavior, mania, or personality changes. Movement disorders included orofacial stereotypies (1/6) and choreoathetosis involving the upper extremities (3/6). Dysautonomia and peripheral nervous system symptoms were absent. Severe neurological dysfunction was present at the peak of the disease (mRS range 3-4). One patient (patient 1, Table 1) who developed facial butterfly erythema and oral ulcer showed antinuclear antibodies and antidsDNA antibody positive in serum. In another patient (patient 5, Table 1), obsolete pulmonary tuberculosis was diagnosed due to obsolete lung lesion on chest enhanced CT scan, positive interferon gamma release assay, and positive tuberculin skin test without evidence of active tuberculosis infection.

Focal demyelination lesions of the bilateral frontal lobes in cranial magnetic resonance imaging (MRI) was found in one patient and cranial MRI were normal in other five patients. Chest-abdomen CT-enhanced scan and tumor markers showed no evidence of neoplastic disease. Thyroid function analysis such as anti-thyroglobulin antibody and anti-thyroid peroxidase antibody was normal. Serum and cerebrospinal fluid tests were negative for viruses, bacteria and fungi. CASPR2-IgG were positive in all sera samples but negative in CSF samples (Table $\mathbf{1}$ and Figure 1). Electroencephalogram demonstrated generalized or focal slow waves in 5 patients.

\section{Treatment and Follow-Up}

The first-line immunotherapy strategy in five patients used high-dose intravenous methylprednisolone (IVMP, $20 \mathrm{mg} / \mathrm{kg}$, maximum dose $1,000 \mathrm{mg}$ once daily for $3 \sim 5$ days) combined with intravenous immunoglobulin (IVIG, $1 \mathrm{~g} / \mathrm{kg}$ once daily for 2 days). Prednisone acetate $(1 \mathrm{mg} / \mathrm{kg}$, maximum dose $60 \mathrm{mg}$ daily) were given orally after methylprednisolone treatment. Another patient (patient 2, Table 2) improved significantly after treatment with IVIG and was subsequently given oral prednisone acetate because the guardian refused to use intravenous methylprednisolone. However, 1 month later the patient developed psychiatric symptoms and sleep disturbances again. The repeat examination were negative for anti-CASPR2 antibody and normal for cranial MRI. The condition improved after steroids (IVMP and prednisone acetate) and IVIG therapy. Two patients received second-line immunotherapy with rituximab, mycophenolate mofetil or cyclophosphamide (Table 2). Four patients received other treatment as the following: 2 antiepileptic drugs, 1 anti-tuberculous therapy, and 3 risperidone (Table 2).

Clinical follow-up data were available from all patients (median 14 months, range 7-23months). The vast majority of patients achieve a stable neurological function within half a year of the disease course (median 5.5 months, range 1-9 months) (Table 2).Five patients showed good neurologic response to immunotherapy with a mRS score 0 . One patients had partial response with a mRS score 2 . Prednisone acetate therapy were tapered for 4-6 months, except for one patient who required 

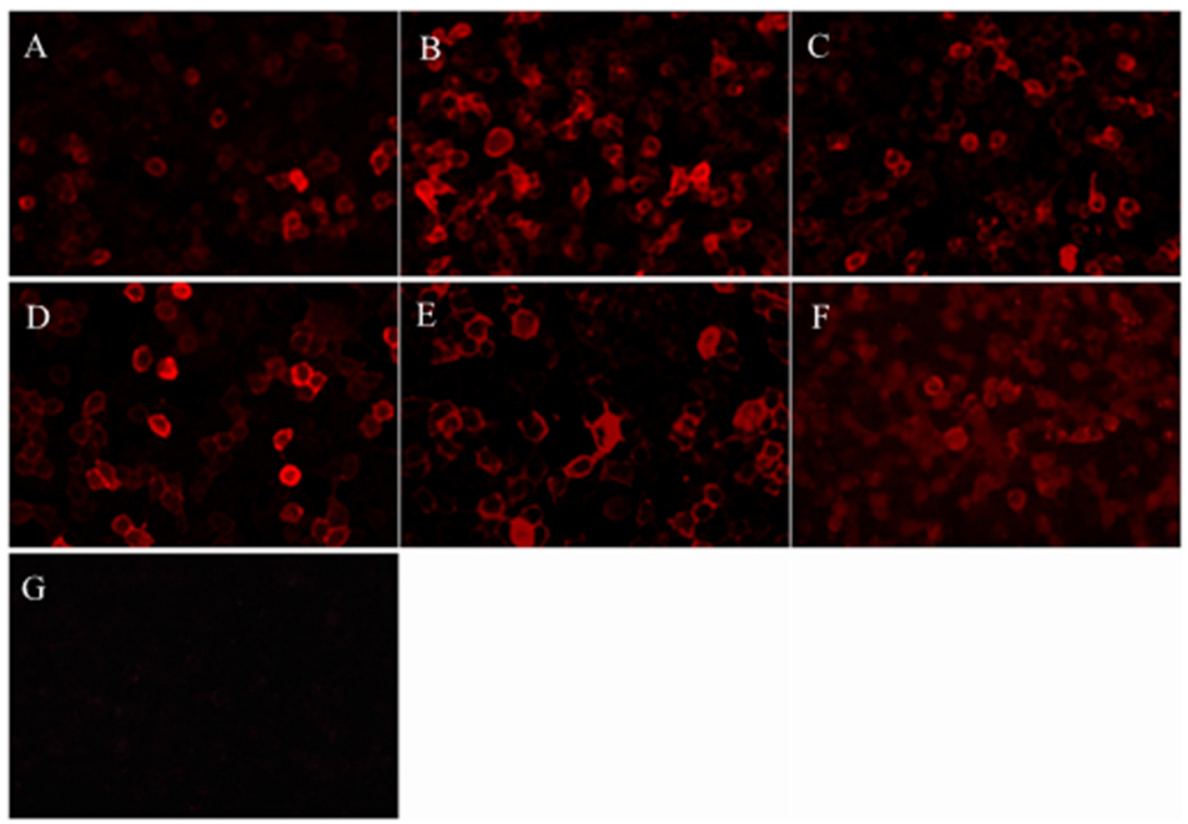

FIGURE 1 | Patients' antibodies reacted with HEK293 cells expressing CASPR2. (A-F) Diluted serum samples of the patient 1-6, respectively, reacted with HEK cells transfected with plasmid encoding human CASPR2 gene. (G) A sample from the control patient shows the negative labeling.

TABLE 2 | Treatment and follow-up of patients with positive anti-CASPR2 antibody.

\begin{tabular}{|c|c|c|c|c|}
\hline \multirow[t]{2}{*}{ No. } & \multicolumn{2}{|c|}{ Therapy } & \multirow[t]{2}{*}{ Final follow-up, months } & \multirow{2}{*}{$\begin{array}{l}\text { mRS score at follow-up } \\
\text { (vs. acute phase)/Time* }\end{array}$} \\
\hline & Immune therapy & AEDs and other therapy & & \\
\hline 1 & IVIG, IVMP, prednisone, CPA & OXC, NZP, LEV & 20 & $0(4) / 1$ \\
\hline 2 & IVIG, IVMP, prednisone & Risperidone & 23 & $0(3) / 9$ \\
\hline 3 & IVIG, IVMP, prednisone & Risperidone & 14 & $0(3) / 6$ \\
\hline 4 & IVIG, IVMP, prednisone & & 13 & $2(3) / 6$ \\
\hline
\end{tabular}

IVIG, intravenous immunoglobulins; IVMP, intravenous methylprednisolone; RTX, rituximab; MMF, mycophenolate mofetil; AEDs, antiepileptic drugs; OXC, oxcarbazepine; NZP, Nitrazepam; LEV, levetiracetam; CPA, cyclophosphamide.

*Time is defined as the time in months to reach the final mRS score during the follow-up period.

\#The patient with an overlap of CASPR2-associated autoimmune encephalitis and systemic lupus erythematosus was given cyclophosphamide infusion therapy for systemic lupus erythematosus.

${ }^{+t}$ The patient was given anti-tuberculous therapy for obsolete pulmonary tuberculosis.

long-term low-dose maintenance for SLE. All patients did not experience recurrence at the time of final follow-up.

\section{DISCUSSION}

The fact that antibodies against VGKC-complex can lead to autoimmune encephalitis was confirmed (7). Hundreds of cases of CASPR2 antibody-associated neurological disorders have been reported in the last decade. A systematic review showed that the most common reported clinical syndromes were autoimmune encephalitis $(69 / 134,51.5 \%)$ and limbic encephalitis (106/274, 38.7\%). Peripheral nerve hyperexcitability (72/191, 37.7\%) and Morvan syndrome (57/251, 22.7\%) were also reported (8). Neurological disorders associated with CASPR2 antibodies occur mainly in adults. Cognitive dysfunction is the most common manifestation in adult patients with CASPR2associated $\mathrm{AE}$, with $<30 \%$ experiencing psychosis and 16$55 \%$ experiencing peripheral hyperexcitability $(2,7,9)$. The proportion of autoimmune encephalitis and peripheral nervous system involvement is lower in children compared to adults. Pediatric patients with a phenotype of pure autoimmune encephalitis are even rarer. A systematic review of LGI1 and CASPR2-related diseases in children showed that $57.1 \%$ of CASPR2 positive patients presented with isolated epilepsy, epileptic encephalopathy or seizure disorder, and only $14.3 \%$ $(2 / 14)$ presented with simple encephalitis. Syndromes with 
mixed central nervous system and peripheral nervous system involvement were $14.3 \%$, with no reports of Morvan syndrome (10). To date, a total of more than 20 cases of CASPR2 autoimmunity in children have been reported, and only a few cases of autoimmune encephalitis have been adequately described $(4,11-14)$. In the present study, all patients had a clinical syndrome of autoimmune encephalitis with no signs of peripheral nerve involvement. Psychotic disorder was the most common symptom, which was observed in 6 patients $(6 / 6)$ in total. More than half of the patients experienced movement disorders, altered consciousness, sleep disturbances or headaches during the course. Seizures occurred in only a minority of patients.

Together with the 2 previously reported patients, $80 \%(8 / 10)$ of pediatric CASPR2 associated AE were older than 8 years of age at onset $(12,13)$. This age distribution feature was not found in MoS which is another common phenotype in children (4). This discrepancy may be due to the small total number of reported cases, and additional clinical data are still needed to summarize the epidemiological features of the disease in the pediatric population. A previous cohort study showed differences in CASPR2 antibody subtypes between patients with $\mathrm{AE}$ and with MoS or NMT. IgG1 antibodies were detected in $58.8 \%$ of all CSF IgG4 antibodies-positive AE patients. IgG1 antibodies were detected in the serum of all AE patients along with IgG4 antibodies in $91.7 \%$ patients. Patients with NMT or MoS had mainly IgG1 subtype in sera $(83.3 \%)$ in association with IgG4 in $41.7 \%$ of the cases (15). Antibody types were not analyzed in pediatric patients. Whether antibody types can be used to explain the differences in clinical phenotypes between age groups needs to be further explored.

The rate of positive cranial MRI in CASPR2 antibodyassociated disease is about $53.1 \%$ (8). In contrast, the positivity rate is about $20-30 \%$ in patients with a clinical spectrum of encephalitis (2). Imaging abnormalities include encephalitis or T2 hyperintensity in medial temporal lobes, hippocampal atrophy, medial temporal lobe sclerosis and hippocampal sclerosis. Cerebellar atrophy has also been reported in isolated cases (7). MRI scans of the pediatric population showed three cases of T2 high signal in the brain, two cases of meningeal enhancement, one case of cauda equina thickening enhancement, and one case of cerebral atrophy $(4,12-14)$. Interestingly, three patients with abnormal brain MRI in a pediatric case series study had negative initial imaging results (4). All patients in our cohort underwent MRI within 4 weeks of onset, and only one patient exhibited focal demyelination of the bilateral frontal lobes. No enhancement scans were performed in all patients and only one patient had a repeat cranial MRI. Therefore, we were unable to clarify the presence of meningeal enhancement or early MRI negativity.

When serum antibody titers are at low levels, there is a risk of false positives $(16,17)$. This was the case in the three patients excluded from this study who presented clinically with transient psychotic symptoms or cerebellar ataxia. In our cohort, $2 / 6$ patients met the diagnostic criteria for pediatric antibodypositive autoimmune encephalitis (5). 4/6 patients did not meet the criteria for paraclinical evidence of neuroinflammation because of the absence of CSF inflammatory changes and MRI features of encephalitis (5). Although serum CASPR2 antibodies were at low titer levels, improvement in the acute phase with immunotherapy and stable neurological status after 1-9 months supported immune-mediated injury. Previous studies have shown an approximately positive rate of $30-40 \%$ for cerebrospinal fluid and $20-30 \%$ for MRI (2). Obtaining brain tissue for biopsy is very difficult in the majority of patients. We prefer that negative cerebrospinal fluid and magnetic resonance examinations in children with CASPR2-associated autoimmune encephalitis should not be used as a basis for a diagnosis of exclusion.

Autoimmune encephalitis has the potential for recurrence. The recurrence rate of anti-N-methyl-D-aspartate receptor encephalitis was ranged from 12 to $29 \%$ (18). Approximately $16-37.5 \%$ of patients with positive anti-CASPR2 antibody experienced a relapse 2 months to several years months after the first episode $(7,9,15)$. In one study, half of the recurrence cases were not treated appropriately in the first episode. Treated patients had a lower relapse rate than untreated patients, which is similar to anti-N-methyl-D-aspartate receptor encephalitis (7). The recurrence rate in Asian adults with anti-CASPR2 encephalitis was $60 \%$, especially higher among the ones treated with corticosteroids alone (19). However, this phenomenon has not been reported in pediatric patients. After a mean follow-up of 13 months all patients had no relapse. Immunotherapy resulted in favorable outcome (mRS 0-2) with control of symptoms. The reason for the recurrence of symptoms in one patient is unknown, and we cannot exclude an association with the non-administration of high-dose methylprednisolone during initial treatment.

Antibody-associated central nervous system autoimmune diseases overlapping systemic lupus erythematosus (SLE) have been reported, but the exact pathogenesis is currently unknown (20-22). One patient presented with rash and positive for multiple autoantibodies during the acute phase of the disease in this study. The patient was diagnosed with overlapping SLE with reference to the SLE diagnostic criteria (23). The symptoms disappeared after treatment with glucocorticoids and intravenous immunoglobulin. Cyclophosphamide infusion therapy was given regularly for the treatment of SLE. In addition, some patients were found to have co-morbid neoplastic diseases including thymoma, prostatic cancer, lung adenocarcinoma, melanoma, endometrial carcinoma, and pancreatic adenocarcinoma. The proportion was more pronounced in adults and in patients with Morvan syndrome and neuromyotonia (7, 8, 15, 24). No tumors were found in our group after detailed examination. To our knowledge, neoplastic diseases were almost exclusively reported in adults.

In the prodromal phase of tuberculosis meningitis, patients experience non-specific signs and symptoms, which include malaise, headache, low-grade fever, and change in personality (25). It is reasonable to suspect tuberculous meningitis in patients with tuberculosis who have psychiatric symptoms. In this study, one patient with obsolete pulmonary tuberculosis was diagnosed with autoimmune encephalitis after detailed 
pathogenetic examination to exclude tuberculous meningitis. This result has implications for the etiological diagnosis of central neurological symptoms in patients with active or obsolete tuberculosis.

\section{LIMITATIONS AND STRENGTHS}

There are some limitations in this study. The relatively short follow-up period and limited number of cases may not reflect the true recurrence rate and also make the analysis of the causes of prognostic differences difficult. When antibody tests show seropositive and cerebrospinal fluid negative, or when clinical presentation does not match the identified antibody, retesting of the sample or use of confirmatory tests (e.g., brain immunohistochemistry or cultured neurons) is recommended (1). Due to the limitations of retrospective studies, we were unable to perform the above validation. The present study provides the largest amount of clinical information to date on CASPR2-associated autoimmune encephalitis in children. It is informative for the study of clinical manifestations in the pediatric phase.

\section{CONCLUSION}

Our study demonstrated the clinical features of CASPR2 antibody associated autoimmune encephalitis in a cohort of pediatric patients. Unlike Morvan syndrome, this type of clinical syndrome seemed to occur more frequently in older children in the present study. Negative cerebrospinal fluid and magnetic resonance examinations are not uncommon in CASPR2-associated autoimmune encephalitis. Most patients had favorable outcomes with aggressive immunotherapy. A few

\section{REFERENCES}

1. Graus F, Titulaer MJ, Balu R, Benseler S, Bien CG, Cellucci T, et al. A clinical approach to diagnosis of autoimmune encephalitis. Lancet Neurol. (2016) 15:391-404. doi: 10.1016/S1474-4422(15)00401-9

2. Bastiaansen AEM, van Sonderen A, Titulaer MJ. Autoimmune encephalitis with anti-leucine-rich glioma-inactivated 1 or anti-contactinassociated protein-like 2 antibodies (formerly called voltage-gated potassium channel-complex antibodies). Curr Opin Neurol. (2017) 30:302-9. doi: 10.1097/WCO.0000000000000444

3. Saint-Martin M, Joubert B, Pellier-Monnin V, Pascual O, Noraz N, Honnorat J. Contactin-associated protein-like 2, a protein of the neurexin family involved in several human diseases. Eur J Neurosci. (2018) 48:190623. doi: 10.1111/ejn.14081

4. Syrbe S, Stettner GM, Bally J, Borggraefe I, Bien CI, Ferfoglia $\mathrm{RI}$, et al. CASPR2 autoimmunity in children expanding to mild encephalopathy with hypertension. Neurology. (2020) 94:e2290301. doi: 10.1212/WNL.0000000000009523

5. Cellucci T, Van Mater H, Graus F, Muscal E, Gallentine W, KleinGitelman MS, et al. Clinical approach to the diagnosis of autoimmune encephalitis in the pediatric patient. Neurol Neuroimmunol Neuroinflamm. (2020) 7:e663. doi: 10.1212/NXI.0000000000000663

6. Zhang L, Wu MQ, Hao ZL, Chiang SM, Shuang K, Lin MT, et al. Clinical characteristics, treatments, and outcomes of patients with anti-N-methyl-daspartate receptor encephalitis: a systematic review of reported cases. Epilepsy Behav. (2017) 68:57-65. doi: 10.1016/j.yebeh.2016.12.019 patients might overlap with other autoimmune diseases such as SLE. Combined tumors in pediatric patients are extremely rare. Further studies with long-term follow-up and large samples are necessary for generalization of epidemiological features, treatment guidance and prognostic assessment.

\section{DATA AVAILABILITY STATEMENT}

The original contributions presented in the study are included in the article/supplementary material, further inquiries can be directed to the corresponding author/s.

\section{ETHICS STATEMENT}

The studies involving human participants were reviewed and approved by Ethics Committee of the Children's Hospital of Chongqing Medical University. Written informed consent to participate in this study was provided by the participants' legal guardian/next of kin.

\section{AUTHOR CONTRIBUTIONS}

CT, YJ, MZ, YH, SH, XL, and LJ contributed to the analysis and interpretation of data and references. CT, YJ, and LJ participated in the conception and writing of the paper. All authors contributed to manuscript revision, read, and approved the submitted version.

\section{ACKNOWLEDGMENTS}

We sincerely thank all the children and their parents for their cooperation during this study.

7. van Sonderen A, Ariño H, Petit-Pedrol M, Leypoldt F, Körtvélyessy P, Wandinger KP, et al. The clinical spectrum of Caspr2 antibody-associated disease. Neurology. (2016) 87:521-8. doi: 10.1212/WNL.0000000000002917

8. Boyko M, Au KLK, Casault C, de Robles P, Pfeffer G. Systematic review of the clinical spectrum of CASPR2 antibody syndrome. J Neurol. (2020) 267:1137-46. doi: 10.1007/s00415-019-09686-2

9. Qin X, Yang H, Zhu F, Wang Q, Shan W. Clinical character of CASPR2 autoimmune encephalitis: a multiple center retrospective study. Front Immunol. (2021) 12:652864. doi: 10.3389/fimmu.2021.652864

10. Nosadini M, Toldo I, Tascini B, Bien CG, Parmeggiani L, De Gaspari P, et al. LGI1 and CASPR2 autoimmunity in children: Systematic literature review and report of a young girl with Morvan syndrome. J Neuroimmunol. (2019) 335:577008. doi: 10.1016/j.jneuroim.2019.577008

11. Hacohen Y, Singh R, Rossi M, Lang B, Hemingway C, Lim M, et al. Clinical relevance of voltage-gated potassium channel-complex antibodies in children. Neurology. (2015) 85:967-75. doi: 10.1212/WNL.0000000000001922

12. Kim SY, Choi SA, Ryu HW, Kim H, Lim BC, Hwang H, et al. Screening autoimmune anti-neuronal antibodies in pediatric patients with suspected autoimmune encephalitis. J Epilepsy Res. (2014) 4:5561. doi: $10.14581 /$ jer. 14012

13. Sunwoo JS, Lee ST, Byun JI, Moon J, Shin JW, Jeong DE, et al. Clinical manifestations of patients with CASPR2 antibodies. J Neuroimmunol. (2015) 281:17-22. doi: 10.1016/j.jneuroim.2015.03.005

14. Lopez-Chiriboga AS, Klein C, Zekeridou A, McKeon A, Dubey D, Flanagan EP, et al. LGI1 and CASPR2 neurological autoimmunity in children. Ann Neurol. (2018) 84:473-80. doi: 10.1002/ana.25310 
15. Joubert B, Saint-Martin M, Noraz N, Picard G, Rogemond V, Ducray F, et al. Characterization of a subtype of autoimmune encephalitis with anticontactin-associated protein-like 2 antibodies in the cerebrospinal fluid, prominent limbic symptoms, and seizures. JAMA Neurol. (2016) 73:111524. doi: 10.1001/jamaneurol.2016.1585

16. Bien CG, Mirzadjanova Z, Baumgartner C, Onugoren MD, Grunwald T, Holtkamp M, et al. Anti-contactin-associated protein-2 encephalitis: relevance of antibody titres, presentation and outcome. Eur J Neurol. (2017) 24:175-86. doi: 10.1111/ene.13180

17. Bien CG. Overinterpretation and overtreatment of low-titer antibodies against contactin-associated protein-2. Front Immunol. (2018) 9:703. doi: 10.3389/fimmu.2018.00703

18. Huang Q, Xie Y, Hu Z, Tang X. Anti-N-methyl-D-aspartate receptor encephalitis: a review of pathogenic mechanisms, treatment, prognosis. Brain Res. (2020) 1727:146549. doi: 10.1016/j.brainres.2019.146549

19. Ghimire P, Khanal UP, Gajurel BP, Karn R, Rajbhandari R, Paudel S, et al. AntiLGI1, anti-GABABR, and anti-CASPR2 encephalitides in Asia: a systematic review. Brain Behav. (2020) 10:e01793. doi: 10.1002/brb3.1793

20. Castro A, Romeu JC, Geraldes R, Pereira da Silva JA. Encephalopathy with upper body hypertonia and myoclonus in patient with systemic lupus erythematosus and anti-CASPR2. Lupus. (2017) 26:84-7. doi: 10.1177/0961203316657431

21. Probstel AK, Thanei M, Erni B, Lecourt AC, Branco L, Andre R, et al. Association of antibodies against myelin and neuronal antigens with neuroinflammation in systemic lupus erythematosus. Rheumatology. (2019) 58:908-13. doi: 10.1093/rheumatology/key282

22. Zhang S, Yang Y, Long T, Li Z. Systemic lupus erythematosus associated with recurrent anti-NMDA receptor encephalitis during pregnancy. Arch Womens Ment Health. (2021) 24:525-8. doi: 10.1007/s00737-020-01088-y
23. Smith EMD, Sen ES, Pain CE. Diagnosis and treatment of childhood-onset systemic lupus erythematosus (European evidence-based recommendations from the SHARE initiative). Arch Dis Child Educ Pract Ed. (2019) 104:25964. doi: 10.1136/archdischild-2017-314049

24. Irani SR, Pettingill P, Kleopa KA, Schiza N, Waters P, Mazia C, et al. Morva0n syndrome: clinical and serological observations in 29 cases. Ann Neurol. (2012) 72:241-55. doi: 10.1002/ana.23577

25. Arshad A, Dayal S, Gadhe R, Mawley A, Shin K, Tellez D, et al. Analysis of tuberculosis meningitis pathogenesis, diagnosis, and treatment. J Clin Med. (2020) 9:2962. doi: 10.3390/jcm9092962

Conflict of Interest: The authors declare that the research was conducted in the absence of any commercial or financial relationships that could be construed as a potential conflict of interest.

Publisher's Note: All claims expressed in this article are solely those of the authors and do not necessarily represent those of their affiliated organizations, or those of the publisher, the editors and the reviewers. Any product that may be evaluated in this article, or claim that may be made by its manufacturer, is not guaranteed or endorsed by the publisher.

Copyright (C) 2021 Tan, Jiang, Zhong, Hu, Hong, Li and Jiang. This is an open-access article distributed under the terms of the Creative Commons Attribution License (CC $B Y)$. The use, distribution or reproduction in other forums is permitted, provided the original author(s) and the copyright owner(s) are credited and that the original publication in this journal is cited, in accordance with accepted academic practice. No use, distribution or reproduction is permitted which does not comply with these terms. 